\title{
Cholecalciferol improves glycemic control in type 2 diabetic patients: a 6-month prospective interventional study
}

This article was published in the following Dove Press journal:

Therapeutics and Clinical Risk Management

7 July 2017

Number of times this article has been viewed

\author{
Aml Mohamed Nada' \\ Dalia A Shaheen ${ }^{2}$ \\ 'Faculty of Medicine, Department \\ of Internal Medicine, ${ }^{2}$ Faculty of \\ Medicine, Department of Medical \\ Biochemistry, Mansoura University, \\ Mansoura, Egypt
}

Background and purpose: To investigate the effects of vitamin D supplementation on glucose homeostasis and lipid profile in type 2 diabetic patients who have vitamin D deficiency.

Patients and methods: One hundred twenty-five type 2 diabetic patients taking oral hypoglycemic agents as mono- or combination therapy were recruited from the diabetes and endocrinology clinic. Subject demographics, duration of diabetes, antidiabetic medication, body mass index (BMI), pulse, and blood pressure (BP) were assessed. Laboratory measurements of serum vitamin D3 level, hemoglobin A1c (HbA1c), fasting plasma glucose (FPG), and lipid profile were measured. Homeostatic model assessment-insulin resistance (HOMA-IR) was calculated whenever fasting insulin (FI) was available. Forty-one patients ( 27 males and 14 females) were started on cholecalciferol replacement $-45,000$ units once weekly for 8 weeks and then 22,500 units once weekly for 16 weeks. Calcium carbonate tablets $500 \mathrm{mg}$ once daily were also prescribed for the initial 2 months of treatment. Measured variables were reassessed after 6 months of replacement therapy. During the trial, subjects were instructed not to change their diabetes drugs or lifestyle.

Results: No significant association was found between vitamin D3 level and any of the measured variables apart from a significant positive correlation with blood urea nitrogen. Vitamin D3 replacement was associated with a significant increase in its level (14.0 $44.0 \mathrm{vs} 31.0$ vs $7.9 \mathrm{ng} / \mathrm{mL}, P<0.001)$. This was associated with a significant reduction of $\mathrm{HbA1c}(7.9 \pm 1.7 \mathrm{vs}$ $7.4 \% \pm 1.2 \%, P=0.001)$ and FPG $(9.1 \pm 4.3$ vs $7.9 \pm 2.4 \mathrm{mmol} / \mathrm{L}, P=0.034)$. Mean reduction of $\mathrm{HbA} 1 \mathrm{c}$ was $0.54 \%$ and that of FPG was $1.22 \mathrm{mmol} / \mathrm{L}$. FI, c-peptide and insulin resistance (IR) were reduced but this was statistically insignificant $(P=0.069,0.376,0.058$, respectively). FI decreased by $22 \%$, HOMA-IR by $27.6 \%$, and c-peptide by $1.83 \%$. Total cholesterol, low-density lipoprotein cholesterol, parathyroid hormone, alkaline phosphatase, serum creatinine, and pulse rate significantly decreased $(4.3 \pm 0.9$ vs $4.0 \pm 0.9 \mathrm{mmol} / \mathrm{L}, P=0.036 ; 2.5 \pm 0.8$ vs $2.2 \pm 0.8 \mathrm{mmol} / \mathrm{L}$, $P=0.018 ; 4.6 \pm 2.1$ vs $3.5 \pm 1.8 \mathrm{pmol} / \mathrm{L}, P=0.001 ; 82.1 \pm 26.2$ vs $66.2 \pm 19.5 \mathrm{U} / \mathrm{L}, P<0.001 ; 74.6 \pm 15.6$ vs $70.7 \pm 14.7 \mu \mathrm{mol} / \mathrm{L}, P=0.047$; and $81.6 \pm 11.9$ vs $77.5 \pm 12.0 \mathrm{bpm}, P=0.045$, respectively). Triglycerides and high-density lipoprotein cholesterol, both systolic and diastolic BP, and BMI did not show significant change.

Conclusion: Cholecalciferol helps improve blood glucose control and cholesterol profile in vitamin D3-deficient type 2 diabetic patients.

Keywords: vitamin D, type 2 diabetes, HbA1c, cholesterol, creatinine, parathyroid hormone

\section{Introduction}

Recently, type 2 diabetes mellitus (T2DM) is considered as one of the nonskeletal diseases associated with vitamin D deficiency. ${ }^{1}$ Both T2DM and vitamin D deficiency have similar risk factors, such as obesity, aging, and sedentary lifestyle. ${ }^{2}$ Cardiovascular
Correspondence: Aml Mohamed Nada Faculty of Medicine, Department of Internal Medicine, Mansoura University, El Gomhouria St, Mansoura, Dakahlia 35516, Egypt

Emailaml_nadanoha@yahoo.com
Therapeutics and Clinical Risk Management 2017:13 8/3-820

813 
diseases (CVDs) and metabolic syndrome disorders are also associated with vitamin D deficiency. ${ }^{3}$

Vitamin D plays a functional role in glucose homeostasis through its effects on insulin secretion and sensitivity. ${ }^{4}$ It may reduce insulin resistance (IR) indirectly through its effect on calcium and phosphate metabolism and through upregulation of the insulin receptor gene. ${ }^{5}$

There are several postulated mechanisms to explain the association between vitamin D deficiency and CVD, such as IR, secondary hyperparathyroidism, and inflammation. ${ }^{6}$ Regulation of the lipid profile is also one of the proposed mechanisms. ${ }^{7}$

Dyslipidemia is a well-described independent risk factor for CVD. High 25-hydroxyvitamin D levels are associated with a favorable lipid profile, whereas low levels are associated with atherogenic serum lipids, as shown in some observational studies. ${ }^{8,9}$ However, intervention studies showed controversial results. ${ }^{8}$

The objective of this study was to determine the effect of vitamin D replacement on fasting plasma glucose (FPG), hemoglobin A1c (HbA1c), and lipid profile in vitamin D-deficient type 2 diabetic patients.

\section{Patients and methods}

One hundred twenty-five adults with a mean age of $52.7 \pm$ 10.3 years, a T2DM duration of $6.7 \pm 6.1$ years taking oral hypoglycemic agents (sulfonylurea, metformin, dipeptidyl-peptidase inhibitors, and pioglitazone) as mono- or combination therapy were recruited from the diabetes and endocrinology clinic from March to September, 2015. The study was already approved by Medical Ethics and Research Committee of King Fahd Hospital, Asir province, Saudi Arabia, and written informed consent was obtained from all participants.

Exclusion criteria included pregnancy, lactation, use of drugs affecting the lipid profile or calcium and bone metabolism, endocrinology disorders, such as hypo- or hyperthyroidism and hyperparathyroidism, smoking, insulin injection, use of antiepileptic drugs, and vitamin D or calcium supplementation. Known sarcoidosis, tuberculosis, potential terminal illness, inflammatory bowel disease, liver or kidney disease and malignancy were also excluded.

Subject demographics, such as age, sex, duration of diabetes, and current antidiabetic medication, were recorded. Body mass index (BMI) was calculated as per the standard equation (mass $[\mathrm{kg}] /$ height $\left[\mathrm{m}^{2}\right]$ ). Weight was measured with a digital scale (Seca 701, Seca, Hamburg, Germany) with a calibrated stadiometer. Both the height and the weight were taken at baseline and follow-up. Blood pressure (BP) was assessed using an automated BP device (Dinamap pro 100v2, GE Medical Systems, Freiburg, Germany) with an appropriate cuff size. Two measurements of systolic BP (SBP) and diastolic BP (DBP) were made 5 minutes apart with the lower reading recorded.

Only 41 patients (27 males and 14 females) were followed until the end of the study (some of the patients preferred to continue with their primary health care center nearby, some had some changes in their medicine, others missed follow-up in our clinic). They received cholecalciferol 45,000 units once weekly for 8 weeks, then 22,500 units once weekly for 16 weeks. Calcium carbonate tablets $500 \mathrm{mg}$ once daily were also prescribed for the first 2 months of treatment.

All variables were measured before and after cholecalciferol supplementation. During the study time, all patients were followed and interviewed to confirm that no change in their medicine or lifestyle was made and that they did not develop side effects of treatment and to check adherence as well.

We measured vitamin D3 level, HbA1c, FPG, and lipid profile for all patients. Other laboratory parameters such as alkaline phosphatase, serum creatinine, blood urea nitrogen (BUN), serum calcium, phosphorus, and fasting insulin (FI) were measured whenever possible (because of availability in the laboratory and financial reasons). IR was calculated by homeostatic model assessment-insulin resistance (HOMA-IR) when FI was available. Measurements were performed in the beginning and at the end of the study.

Blood samples were collected from all participants, after an overnight fasting and left to clot. Sera were separated by centrifuging blood at 3,000 rpm for 15 minutes and stored at $-20^{\circ} \mathrm{C}$ until analysis. Sera for lipids were centrifuged for 10 minutes at 3,000 rpm, for collection of the aqueous phase.

Vitamin D3 was measured using the $25(\mathrm{OH})$ vitamin D3 ELISA Kit (Immundiagnostik, Bensheim and Biomedica, Vienna, Austria) according to the manufacturer's instruction using a sunrise plate reader at $450 \mathrm{~nm}$ against $620 \mathrm{~nm}$ as a reference. ${ }^{10}$

Total cholesterol (TC), high-density lipoprotein cholesterol (HDL-C), and TG were measured by BioMerieux Laboratory, Marcy 1'Etoile, France. ${ }^{11,12}$ Low-density lipoprotein cholesterol (LDL-C) was calculated according to the Friedewald formula. ${ }^{13}$ Serum glucose was determined colorimetrically according to the method of Trinder. ${ }^{14}$ HbA1c was measured immunoturbidimetrically (using COBAS INTEGTRA 400 machine; Roche Diagnostics, Indianapolis, IN, USA). The final result was expressed as $\mathrm{HbAlc}$ percent and is calculated from the $\mathrm{HbA} 1 \mathrm{c} / \mathrm{Hb}$ 
ratio, including a conversion equation to match a high performance liquid chromatography reference method. HbA1c $(\%)=\mathrm{HbA} 1 \mathrm{c} / \mathrm{Hb} \times 175.8+1.73 .{ }^{15}$

Calcium, phosphate, and alkaline phosphatase were assessed colorimetrically using calcium detection kit (product number ab102505), phosphate assay kit (product number ab65622), and alkaline phosphatase kit (product number ab83369) purchased from Abcam (Cambridge, UK). Gamma glutamyl transferase was measured using Reflotron Plus Analyzer and Roche kits (Roche Diagnostics GmbH, Mannheim, Germany).

The ELISA machine was used to read the absorbance in the samples. Vitamin D deficiency was defined as a serum level of $25(\mathrm{OH}) \mathrm{D}<20 \mathrm{ng} / \mathrm{mL}$ and insufficiency for a level of 20-29 ng/mL. ${ }^{16}$ HOMA-IR was calculated based on the formula: HOMA-IR $=$ FPG $(\mathrm{mmol} / \mathrm{L}) \times$ insulin $(\mu \mathrm{Iu} / \mathrm{mL}) / 22.5 \cdot{ }^{17}$

\section{Statistical analysis}

Data were analyzed using the Statistical Package for Social Sciences (IBM SPSS, version 20). Descriptive statistics for variables were calculated (ie, frequency, mean, and standard deviation). Tests of significance were applied, for example, paired $t$-test and Pearson's correlation coefficient. $P$-values $<0.5$ were considered as statistically significant.

\section{Results}

The study included 125 diabetic patients (57 women and $68 \mathrm{men}$ ) aged $52.7 \pm 10.3$ years, with a BMI of $33.0 \pm 6.1 \mathrm{~kg} / \mathrm{m}^{2}$ and a diabetes duration of $6.7 \pm 6.1$ years (Table 1).

Eighteen patients (14.4\%) had vitamin D insufficiency (20-30 ng/mL) and 107 patients $(85.6 \%)$ had vitamin D deficiency (level $<20 \mathrm{ng} / \mathrm{mL}$ ). No significant association was found between vitamin $\mathrm{D}$ level and any measured variables apart from the significant positive correlation with BUN (Table 2).

Vitamin D3 replacement was associated with a significant increase in its level $(14.0 \pm 4.0$ vs $31.0 \pm 7.9, P<0.001)$ with all 41 cases achieving a level $>20 \mathrm{ng} / \mathrm{mL}$ and $63 \%$ of patients achieving a level $>30 \mathrm{ng} / \mathrm{mL}$. This was associated with a significant reduction of $\mathrm{HbA1c}(7.9 \pm 1.7$ vs $7.4 \pm 1.2, P=0.001)$ and FPG $(9.1 \pm 4.3$ vs $7.9 \pm 2.4, P=0.034)$. Mean reduction of $\mathrm{HbA} 1 \mathrm{c}$ was $0.54 \%$ and that of FPG was $1.2 \mathrm{mmol} / \mathrm{L}$. FI, c-peptide, and IR decreased, but this was statistically insignificant $(P=0.069$, 0.376 , and 0.058 , respectively). FI was reduced by $22 \%$, IR by $27.6 \%$, and c-peptide by $1.8 \%$ (Table 3 ; Figure 1 ).

Both TC and LDL-C significantly decreased $(4.3 \pm 0.9$ vs $4.0 \pm 0.9, P=0.036$ and $2.5 \pm 0.8$ vs $2.2 \pm 0.8, P=0.018$,
Table I Personal characteristics and baseline laboratory parameters of the study sample

\begin{tabular}{|c|c|c|}
\hline Variables & $\begin{array}{l}\text { Number } \\
\text { of patients }\end{array}$ & Mean \pm SD \\
\hline Age, years & 125 & $52.7 \pm 10.3$ \\
\hline Duration of DM, years & 125 & $7.7 \pm 6.1$ \\
\hline $\mathrm{BW}, \mathrm{kg}$ & 125 & $84.9 \pm 14.5$ \\
\hline Height, $m$ & 125 & $160.9 \pm 9.0$ \\
\hline BMI, $\mathrm{kg} / \mathrm{m}^{2}$ & 125 & $33.0 \pm 6.1$ \\
\hline $\mathrm{SBP}, \mathrm{mmHg}$ & 120 & $126.1 \pm 15.1$ \\
\hline $\mathrm{DBP}, \mathrm{mmHg}$ & 120 & $73.9 \pm 8.7$ \\
\hline Pulse, ppm & 117 & $81.2 \pm 12.2$ \\
\hline Vitamin D3, ng/mL & 125 & $\mid 4.1 \pm 4.7$ \\
\hline $\mathrm{FPG}, \mathrm{mmol} / \mathrm{L}$ & 125 & $8.3 \pm 3.3$ \\
\hline $\mathrm{HbAlc}$ & 125 & $7.8 \pm 1.5$ \\
\hline $\mathrm{FI}, \mathrm{uU} / \mathrm{mL}$ & 57 & $11.6 \pm 6.5$ \\
\hline c-peptide, $\mathrm{ng} / \mathrm{mL}$ & 52 & $2.8 \pm 0.8$ \\
\hline HOMA-IR & 57 & $4.1 \pm 3.0$ \\
\hline PTH, pmol/L & 79 & $5.4 \pm 2.5$ \\
\hline $\mathrm{Ca}, \mathrm{mmol} / \mathrm{L}$ & 101 & $2.3 \pm 0.1$ \\
\hline $\mathrm{P}, \mathrm{mmol} / \mathrm{L}$ & 94 & $1.2 \pm 0.2$ \\
\hline AP, units/L & 113 & $85.4 \pm 31.3$ \\
\hline $\mathrm{TC}, \mathrm{mmol} / \mathrm{L}$ & 125 & $4.2 \pm 1.0$ \\
\hline $\mathrm{LDL}-\mathrm{C}, \mathrm{mmol} / \mathrm{L}$ & 123 & $1.1 \pm 0.3$ \\
\hline $\mathrm{HDL}-\mathrm{C}, \mathrm{mmol} / \mathrm{L}$ & 123 & $1.1 \pm 0.3$ \\
\hline $\mathrm{TG}, \mathrm{mmol} / \mathrm{L}$ & 124 & $2.7 \pm 13.4$ \\
\hline Creatinine, $\mu \mathrm{mol} / \mathrm{L}$ & 119 & $71.6 \pm 17.7$ \\
\hline $\mathrm{BUN}, \mathrm{mmol} / \mathrm{L}$ & 120 & $4.4 \pm 0.3$ \\
\hline $\mathrm{Na}, \mathrm{mmol} / \mathrm{L}$ & 118 & $138.5 \pm 2.9$ \\
\hline $\mathrm{K}, \mathrm{mmol} / \mathrm{L}$ & 120 & $4.4 \pm 0.3$ \\
\hline $\mathrm{Mg}, \mathrm{mmol} / \mathrm{L}$ & 51 & $0.8 \pm 0.1$ \\
\hline GGT, units/L & 78 & $41.3 \pm 30.8$ \\
\hline RPP & 117 & $10,252.2 \pm 1,985.6$ \\
\hline
\end{tabular}

Abbreviations: AP, alkaline phosphatase; BMI, body mass index; BUN, blood urea nitrogen; BW, body weight; D3, vitamin D3; DBP, diastolic blood pressure; FI, fasting insulin; FPG, fasting plasma glucose; GGT, gamma glutamate transferase; $\mathrm{HbAlc}$, hemoglobin Alc; HDL-C, high-density lipoprotein cholesterol; HOMAIR, homeostatic model assessment-Insulin resistance; LDL-C, low-density lipoprotein cholesterol; PTH, parathyroid hormone; RPP, rate pressure product; SBP, systolic blood pressure; TC, total cholesterol; TG, triglyceride; SD, standard deviation.

respectively). Triglycerides and HDL-C did not show significant changes ( $P=0.874$ and 0.444 , respectively).

Parathyroid hormone (PTH) and alkaline phosphatase significantly decreased with cholecalciferol therapy (4.6 \pm 2.1 vs $3.5 \pm 1.8, P=0.001$ and $82.1 \pm 26.2$ vs $66.2 \pm 19.5, P<0.001$, respectively). There was no change in serum calcium level, but serum phosphate level significantly increased $(P=0.75,0.02$, respectively). Interestingly, serum creatinine significantly decreased ( $74.6 \pm 15.6$ vs $70.7 \pm 14.7, P=0.047$ ). Pulse rate was significantly reduced ( $81.6 \pm 11.9$ vs $77.5 \pm 12.0, P=0.045)$. There was a trend toward the reduction of SBP, but this did not reach a statistical significance $(P=0.081)$. No significant change in DBP or BMI was noticed (Table 3; Figure 2). 
Table 2 Correlation between initial serum levels of vitamin D3 and measured laboratory variables

\begin{tabular}{|c|c|c|c|}
\hline $\begin{array}{l}\text { Laboratory } \\
\text { variables }\end{array}$ & $\begin{array}{l}\text { Number } \\
\text { of patients }\end{array}$ & $\begin{array}{l}\text { Pearson's } \\
\text { correlation } \\
\text { coefficient, } r\end{array}$ & $P$-value \\
\hline $\mathrm{FPG}, \mathrm{mmol} / \mathrm{L}$ & 125 & -0.04 & 0.64 \\
\hline $\mathrm{HbAlc}$ & 125 & 0.03 & 0.739 \\
\hline $\mathrm{Fl}, \mathrm{uU} / \mathrm{mL}$ & 57 & -0.09 & 0.519 \\
\hline c-peptide, $\mathrm{ng} / \mathrm{mL}$ & 52 & -0.14 & 0.331 \\
\hline HOMA-IR & 57 & -0.13 & 0.331 \\
\hline PTH, pmol/L & 79 & 0.02 & 0.878 \\
\hline $\mathrm{Ca}, \mathrm{mmol} / \mathrm{L}$ & 101 & 0.15 & 0.142 \\
\hline $\mathrm{P}, \mathrm{mmol} / \mathrm{L}$ & 94 & -0.05 & 0.638 \\
\hline$A P$, units/L & 113 & -0.09 & 0.373 \\
\hline $\mathrm{TC}, \mathrm{mmol} / \mathrm{L}$ & 124 & -0.07 & 0.445 \\
\hline LDL-C, mmol/L & 123 & -0.14 & 0.119 \\
\hline $\mathrm{HDL}-\mathrm{C}, \mathrm{mmol} / \mathrm{L}$ & 122 & 0.03 & 0.714 \\
\hline $\mathrm{TG}, \mathrm{mmol} / \mathrm{L}$ & 123 & 0.15 & 0.094 \\
\hline Creatinine, $\mu \mathrm{mol} / \mathrm{L}$ & 119 & 0.10 & 0.301 \\
\hline $\mathrm{BUN}, \mathrm{mmol} / \mathrm{L}$ & 120 & 0.19 & 0.036 \\
\hline $\mathrm{Na}, \mathrm{mmol} / \mathrm{L}$ & 118 & -0.10 & 0.294 \\
\hline $\mathrm{K}, \mathrm{mmol} / \mathrm{L}$ & 120 & -0.01 & 0.909 \\
\hline $\mathrm{Mg}, \mathrm{mmol} / \mathrm{L}$ & 51 & 0.16 & 0.250 \\
\hline GGT, units/L & 78 & 0.03 & 0.789 \\
\hline
\end{tabular}

Abbreviations: AP, alkaline phosphatase; BUN, blood urea nitrogen; Fl, fasting insulin; FPG, fasting plasma glucose; GGT, gamma glutamate transferase; HbAlc, hemoglobin Alc; HDL-C, high-density lipoprotein cholesterol; HOMA-IR, homeostatic model assessment-Insulin resistance; LDL-C, low-density lipoprotein cholesterol; PTH, parathyroid hormone; TC, total cholesterol; TG, triglyceride.

It was also found that the higher the initial vitamin D3 level, the more the change of FI, HOMA-IR, and c-peptide. There was no significant association between basal vitamin D3 and the change in FPG, HbA1c, TC, and LDL-C after replacement. Also the higher the baseline values of FPG, HbA1c, FI, creatinine, LDL-C, and TC, the smaller the reduction of vitamin $\mathrm{D}$ intake. We could not find a significant correlation between the reduction in PTH and the change in FPG, HbA1c, FI, HOMA-IR, c-peptide, TC, or LDL-C. A significant and direct association between the reduction of alkaline phosphatase and the decrease in both FPG and HbA1c was noticed $(P=0.001$ and 0.001 , respectively). There was a negative correlation between the change in PTH level and the reduction in serum creatinine; the more the decrease in $\mathrm{PTH}$, the less the decrease in creatinine $(P=0.049)$ (Tables 4-8; Figure 3).

\section{Discussion}

Similar to our study in Saudi type 2 diabetic subjects, AlDaghri et al found a positive effect of vitamin D intake on HbA1c and insulin sensitivity based on HOMA-IR and HOMA- $\beta .^{18}$ There was also a significant decrease in LDL-C and $\mathrm{TC}$ without changes in TG or HDL-C, although only
Table 3 Changes in different variables with vitamin D3 replacement

\begin{tabular}{|c|c|c|c|c|}
\hline Variables & $\begin{array}{l}\text { Number } \\
\text { of patients }\end{array}$ & $\begin{array}{l}\text { Before } \\
\text { treatment, } \\
\text { mean } \pm \text { SD }\end{array}$ & $\begin{array}{l}\text { After } \\
\text { treatment, } \\
\text { mean } \pm S D\end{array}$ & $P$-value \\
\hline BW, kg & 41 & $85.1 \pm 12.4$ & $85.1 \pm 13.3$ & 0.944 \\
\hline $\mathrm{BMI}, \mathrm{kg} / \mathrm{m}^{2}$ & 41 & $32.3 \pm 4.8$ & $32.5 \pm 4.6$ & 0.604 \\
\hline $\mathrm{SBP}, \mathrm{mmHg}$ & 41 & $123.0 \pm 15.5$ & $119.6 \pm 13.9$ & 0.081 \\
\hline $\mathrm{DBP}, \mathrm{mmHg}$ & 41 & $72.9 \pm 8.6$ & $71.6 \pm 8.8$ & 0.211 \\
\hline Pulse, ppm & 41 & $81.6 \pm 11.9$ & $77.5 \pm 12.0$ & 0.045 \\
\hline Vitamin D3, ng/mL & 41 & $14.0 \pm 4.0$ & $31.0 \pm 7.9$ & $<0.001$ \\
\hline FPG, $\mathrm{mmol} / \mathrm{L}$ & 41 & $9.1 \pm 4.3$ & $7.9 \pm 2.4$ & 0.034 \\
\hline $\mathrm{HbAlc}$ & 41 & $7.9 \pm 1.8$ & $7.4 \pm 1.2$ & 0.001 \\
\hline $\mathrm{FI}, \mathrm{uU} / \mathrm{mL}$ & 12 & $12.9 \pm 7.6$ & $8.5 \pm 4.1$ & 0.069 \\
\hline c-peptide, $\mathrm{ng} / \mathrm{mL}$ & 12 & $2.7 \pm 0.8$ & $2.6 \pm 0.5$ & 0.376 \\
\hline HOMA-IR & 12 & $4.7 \pm 3.5$ & $2.6 \pm 1.1$ & 0.058 \\
\hline PTH, pmol/L & 20 & $4.6 \pm 2.1$ & $3.5 \pm 1.8$ & 0.001 \\
\hline $\mathrm{Ca}, \mathrm{mmol} / \mathrm{L}$ & 27 & $2.3 \pm 0.1$ & $2.3 \pm 0.1$ & 0.752 \\
\hline $\mathrm{P}, \mathrm{mmol} / \mathrm{L}$ & 24 & $1.1 \pm 0.2$ & $1.2 \pm 0.2$ & 0.022 \\
\hline$A P$, units $/ L$ & 32 & $82.1 \pm 26.2$ & $66.2 \pm 19.5$ & $<0.001$ \\
\hline $\mathrm{TC}, \mathrm{mmol} / \mathrm{L}$ & 41 & $4.3 \pm 0.9$ & $4.0 \pm 0.9$ & 0.036 \\
\hline LDL-C, mmol/L & 41 & $2.5 \pm 0.8$ & $2.2 \pm 0.8$ & 0.018 \\
\hline $\mathrm{HDL}-\mathrm{C}, \mathrm{mmol} / \mathrm{L}$ & 41 & $1.2 \pm 0.4$ & $1.2 \pm 0.4$ & 0.444 \\
\hline $\mathrm{TG}, \mathrm{mmol} / \mathrm{L}$ & 41 & $1.5 \pm 0.7$ & $1.4 \pm 0.9$ & 0.874 \\
\hline Creatinine, $\mu \mathrm{mol} / \mathrm{L}$ & 38 & $74.6 \pm 15.6$ & $70.7 \pm 14.7$ & 0.047 \\
\hline BUN, mmol/L & 38 & $5.3 \pm 1.4$ & $5.0 \pm 1.5$ & 0.223 \\
\hline $\mathrm{Na}, \mathrm{mmol} / \mathrm{L}$ & 36 & $138.5 \pm 2.8$ & $138.3 \pm 3.1$ & 0.616 \\
\hline $\mathrm{K}, \mathrm{mmol} / \mathrm{L}$ & 38 & $4.6 \pm 0.3$ & $4.5 \pm 0.4$ & 0.152 \\
\hline
\end{tabular}

Abbreviations: AP, alkaline phosphatase; BMI, body mass index; BUN, blood urea nitrogen; BW, body weight; DBP, diastolic blood pressure; Fl, fasting insulin; FPG, fasting plasma glucose; GGT, gamma glutamate transferase; HbAlc, hemoglobin Alc; HDL-C, high-density lipoprotein cholesterol; HOMA-IR, homeostatic model assessment-Insulin resistance; LDL-C, low-density lipoprotein cholesterol; PTH, parathyroid hormone; SBP, systolic blood pressure; TC, total cholesterol;TG, triglyceride; SD, standard deviation.

a mean increase of serum vitamin D3 was $8 \mathrm{ng} / \mathrm{mL}$ with suboptimal final level. ${ }^{18}$ Our study supports the positive effect of vitamin D supplementation on glycemic control in T2DM and associated dyslipidemia in Saudi patients, although the duration of therapy and regimen of vitamin D supplementation and its mean increase are different.

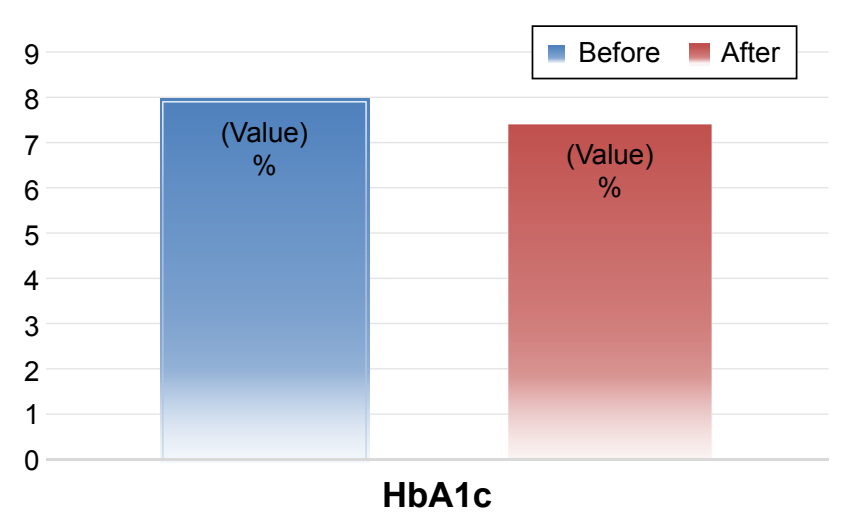

Figure I Change in hemoglobin Alc ( $\mathrm{HbAlc})$ after vitamin D intake. 


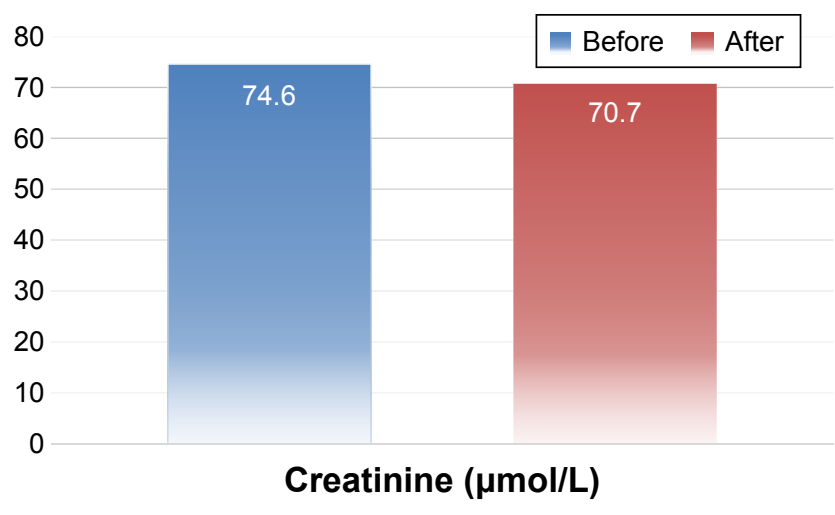

Figure 2 Change of serum creatinine after vitamin D intake.

In the retrospective study conducted by Sabherwal et al on South Asians with established T2DM, patients were treated with combined oral vitamin $\mathrm{D}_{3}$ and calcium for 3 months. Post treatment, a significant decrease in $\mathrm{HbAl} \mathrm{c}$ was achieved in the deficiency and insufficiency groups $(0.70 \% \pm 0.77 \%$, $P<0.001$ and $0.21 \% \pm 0.28 \%, P=0.001$, respectively). The change in weight was only significant in the vitamin D-deficient group. There were also negative correlations between the changes in $\mathrm{HbA} 1 \mathrm{c}$ and weight with the change in vitamin $\mathrm{D}(P<0.05) .{ }^{19}$

In our study, the significant reduction in pulse rate may be explained by the presence of vitamin $\mathrm{D}$ receptors in the t-tubules in the heart which are ideally positioned to exert an immediate effect on signal transduction mediators and ion channels $^{20}$ (Tables 3 and 7).

In Soric et al's study, patients with higher baseline $\mathrm{HbAlc}$ had a significantly greater reduction in $\mathrm{HbA} 1 \mathrm{c}$ on receiving vitamin D for 12 weeks. This reduction was only significant when baseline $\mathrm{HbA} 1 \mathrm{c}$ was $>9 \% .{ }^{21}$ In our study, the higher the baseline values of FPG, HbA1c, FI, HOMA-IR, c-peptide, TC, LDL-C, the smaller the reduction of each.

Table 4 Correlation coefficients between changes in laboratory variables with their initial levels before treatment

\begin{tabular}{lll}
\hline $\begin{array}{l}\text { Laboratory } \\
\text { variables }\end{array}$ & $\begin{array}{l}\text { Pearson's } \\
\text { correlation } \\
\text { coefficient, } \boldsymbol{r}\end{array}$ & P-value \\
\hline $\mathrm{FPG}, \mathrm{mmol} / \mathrm{L}$ & -0.83 & $<0.00 \mathrm{I}$ \\
$\mathrm{HbAlc}$ & -0.77 & $<0.00 \mathrm{I}$ \\
$\mathrm{Fl}, \mathrm{uU} / \mathrm{mL}$ & -0.75 & $0.00 \mathrm{I}$ \\
$\mathrm{c}-$ peptide, $\mathrm{ng} / \mathrm{mL}$ & -0.09 & 0.747 \\
Creatinine, $\mu \mathrm{mol} / \mathrm{L}$ & -0.45 & 0.005 \\
$\mathrm{LDL}-\mathrm{C}, \mathrm{mmol} / \mathrm{L}$ & -0.43 & 0.005 \\
TC, $\mathrm{mmol} / \mathrm{L}$ & -0.42 & 0.007 \\
\hline
\end{tabular}

Abbreviations: $\mathrm{Fl}$, fasting insulin; $\mathrm{FPG}$, fasting plasma glucose; $\mathrm{HbAlc}$, hemoglobin Alc; LDL-C, low-density lipoprotein cholesterol; TC, total cholesterol.
Table 5 Mean percent changes for different laboratory variables

\begin{tabular}{ll}
\hline Laboratory variables & $\begin{array}{l}\text { Mean percent change } \\
\text { for different variables } \\
\text { ([B-A] } \times \text { I 00/A) }\end{array}$ \\
\hline $\mathrm{Fl}, \mathrm{uU} / \mathrm{mL}$ & 22.21 \\
c-peptide, $\mathrm{ng} / \mathrm{mL}$ & 1.83 \\
$\mathrm{HOMA}-\mathrm{IR}$ & 27.58 \\
$\mathrm{TC}, \mathrm{mmol} / \mathrm{L}$ & 4.94 \\
$\mathrm{LDL}-\mathrm{C}, \mathrm{mmol} / \mathrm{L}$ & 8.60 \\
$\mathrm{HDL}-\mathrm{C}, \mathrm{mmol} / \mathrm{L}$ & 1.05 \\
$\mathrm{TG}, \mathrm{mmol} / \mathrm{L}$ & 1.86 \\
$\mathrm{Creatinine}, \mu \mathrm{mol} / \mathrm{L}$ & 3.59
\end{tabular}

Notes: $B$ : the value of the measured variable after vitamin $D$ replacement. A: the initial value of the measured variable before vitamin $D$ replacement.

Abbreviations: FI, fasting insulin; HDL-C, high-density lipoprotein cholesterol; HOMA-IR, homeostatic model assessment-insulin resistance; LDL-C, low-density lipoprotein cholesterol; TC, total cholesterol; TG, triglyceride.

von Hurst et al, in their study, showed improved insulin sensitivity. ${ }^{22}$ However, Talaei et al showed that the effect of vitamin D3 on IR was significant only when its concentration was $40-60 \mathrm{ng} / \mathrm{mL}$, and in lower and upper concentrations, it had no effect. ${ }^{23}$ Thus, the insignificant change in FI and IR in our study may be attributed to the final vitamin D3 level $(31 \mathrm{ng} / \mathrm{mL}$ ) or to the small number of patients for whom these variables were available.

In our study, HOMA-IR decreased by $27.6 \%$ with a mean increase of vitamin D3 of $17 \mathrm{ng} / \mathrm{mL}$, whereas in the study by Sugden et al, HOMA-IR significantly improved with an increase of $\geq 11 \mathrm{nmol} / \mathrm{L}$. However, they did not show a significant change in $\mathrm{HbAlc} .^{24}$

In Shab-Bidar et al's study, there was also a significant improvement in FPG, the Quantitative Insulin Check Index, and HbAlc. ${ }^{25}$ Tabesh et al found that the effect of vitamin D on glucose homeostasis was evident and significant only when it was administered in combination with calcium and not as monotherapy. ${ }^{26}$ In our study, oral calcium carbonate $500 \mathrm{mg}$ was given once daily for the first 2 months of treatment.

In contrary to Chiu et al's report, ${ }^{27}$ we could not find a significant association between the initial vitamin D3 level

Table 6 Mean changes for different laboratory variables

\begin{tabular}{ll}
\hline Laboratory variables & $\begin{array}{l}\text { Mean change }= \\
\text { (before }- \text { after) }\end{array}$ \\
\hline Vitamin D3, ng/mL & 16.99 \\
$\mathrm{FPG}, \mathrm{mmol} / \mathrm{L}$ & 1.22 \\
$\mathrm{HbAlc}$ & 0.54 \\
$\mathrm{TC}, \mathrm{mmol} / \mathrm{L}$ & 0.26 \\
$\mathrm{LDL}-\mathrm{C}, \mathrm{mmol} / \mathrm{L}$ & 0.27 \\
$\mathrm{HDL}-\mathrm{C}, \mathrm{mmol} / \mathrm{L}$ & 0.02 \\
$\mathrm{TG}, \mathrm{mmol} / \mathrm{L}$ & 0.01 \\
\hline
\end{tabular}

Abbreviations: FPG, fasting plasma glucose; $\mathrm{HbAlc}$, hemoglobin A Ic; LDL-C, lowdensity lipoprotein cholesterol; HDL-C, high-density lipoprotein cholesterol; TC, total cholesterol; TG, triglyceride. 
Table 7 Correlation between initial levels of vitamin $D$ and changes in different laboratory variables

\begin{tabular}{lll}
\hline $\begin{array}{l}\text { Laboratory } \\
\text { variables }\end{array}$ & $\begin{array}{l}\text { Pearson's correlation } \\
\text { coefficient, } \boldsymbol{r}\end{array}$ & $\boldsymbol{P}$-value \\
\hline $\mathrm{hFPG}, \mathrm{mmol} / \mathrm{L}$ & 0.29 & 0.074 \\
$\mathrm{HbAlc}$ & 0.07 & 0.661 \\
$\mathrm{Fl}, \mathrm{uU} / \mathrm{mL}$ & 0.33 & 0.036 \\
c-peptide, $\mathrm{ng} / \mathrm{mL}$ & 0.35 & 0.027 \\
$\mathrm{HOMA}-\mathrm{R}$ & 0.37 & 0.018 \\
TC, $\mathrm{mmol} / \mathrm{L}$ & -0.08 & 0.612 \\
LDL-C, mmol/L & 0.07 & $0.67 \mathrm{I}$ \\
Creatinine, $\mu \mathrm{mol} / \mathrm{L}$ & 0.08 & 0.620 \\
\hline
\end{tabular}

Abbreviations: $\mathrm{Fl}$, fasting insulin; $\mathrm{FPG}$, fasting plasma glucose; $\mathrm{HbAlc}$, hemoglobin Alc; HOMA-IR, homeostatic model assessment-Insulin resistance; LDL-C, lowdensity lipoprotein cholesterol; TC, total cholesterol.

and FPG, A1c, FI, HOMA-IR, or lipid profile. Vitamin D effects on insulin sensitivity and diabetes may be explained by the presence of vitamin $D$ receptors on pancreatic $\beta$ cells ${ }^{1}$ and skeletal muscle, ${ }^{28}$ and by the presence of vitamin D response in the insulin gene. ${ }^{5,29}$ Vitamin D attenuates the expression of proinflammatory cytokines involved in IR. ${ }^{5,30}$ It also increases intracellular calcium, thus increasing glucose transport into the cells. ${ }^{31}$ It regulates nuclear peroxisome proliferative-activated receptor that plays a

Table 8 The correlation coefficients between parathyroid hormone, alkaline phosphatase and phosphate levels with different laboratory variables

\begin{tabular}{|c|c|c|c|}
\hline Laboratory variables & PTH & $\mathbf{A P}$ & $\mathbf{P}$ \\
\hline \multicolumn{4}{|l|}{$\mathrm{FPG}, \mathrm{mmol} / \mathrm{L}$} \\
\hline$r$ & -0.18 & 0.56 & 0.05 \\
\hline$P$-value & 0.436 & 0.001 & 0.804 \\
\hline \multicolumn{4}{|l|}{$\mathrm{HbAlc}$} \\
\hline$r$ & -0.04 & 0.45 & 0.27 \\
\hline$P$-value & 0.885 & 0.011 & 0.211 \\
\hline \multicolumn{4}{|l|}{$\mathrm{TC}, \mathrm{mmol} / \mathrm{L}$} \\
\hline$r$ & 0.02 & 0.11 & -0.31 \\
\hline$P$-value & 0.938 & 0.534 & 0.138 \\
\hline \multicolumn{4}{|l|}{ LDL-C, mmol/L } \\
\hline$r$ & 0.01 & 0.16 & -0.22 \\
\hline$P$-value & 0.953 & 0.394 & 0.310 \\
\hline$r$ & -0.45 & -0.15 & -0.07 \\
\hline$P$-value & 0.049 & 0.414 & 0.759 \\
\hline \multicolumn{4}{|l|}{$\mathrm{BUN}, \mathrm{mmol} / \mathrm{L}$} \\
\hline$r$ & 0.43 & -0.05 & -0.00 \\
\hline$P$-value & 0.061 & 0.789 & 0.992 \\
\hline \multicolumn{4}{|l|}{$\mathrm{FI}, \mathrm{uU} / \mathrm{mL}$} \\
\hline$r$ & -0.17 & -0.168 & 0.133 \\
\hline$P$-value & 0.484 & 0.359 & 0.535 \\
\hline \multicolumn{4}{|l|}{ c-peptide, $n g / m L$} \\
\hline$r$ & -0.22 & -0.04 & 0.02 \\
\hline$P$-value & 0.361 & 0.827 & 0.910 \\
\hline
\end{tabular}

Abbreviations: PTH, parathyroid hormone; AP, alkaline phosphatase; P, phosphate; BUN, blood urea nitrogen; Fl, fasting insulin; FPG, fasting plasma glucose; HbAlc, hemoglobin Alc; LDL-C, low-density lipoprotein cholesterol; PTH, parathyroid hormone; TC, total cholesterol; TG, triglyceride.

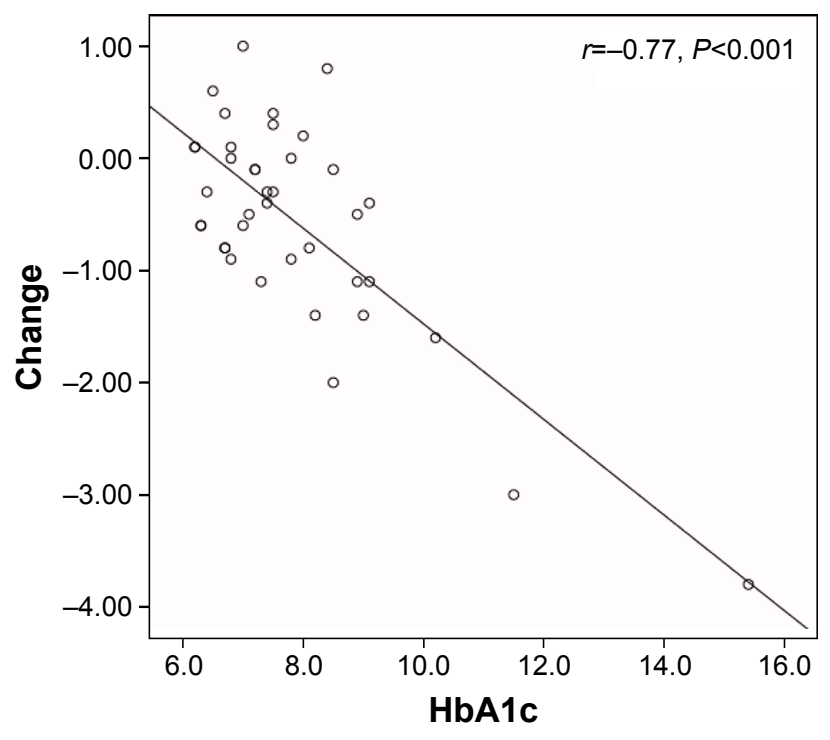

Figure 3 Change in hemoglobin $\mathrm{Alc}(\mathrm{HbAlc})$ in relation with its initial level before treatment.

great role in insulin sensitivity. ${ }^{32}$ Correction of secondary hyperparathyroidism is another suggested mechanism of action..$^{33}$ In our study, there was a significant reduction in PTH and alkaline phosphatase with an increase in phosphate level.

In the randomized controlled trials that were published until 2015, Jafari et al found that vitamin D significantly reduced serum TC and LDL-C in patients with T2DM, but serum TG and HDL-C did not show significant changes. Baseline vitamin D3, its dosage, intervention duration, and the method of its intake influence the effect on lipid markers. ${ }^{34}$ Our study results are in complete concordance with the results of this meta-analysis.

Shab-Bidar et al also observed a positive effect of vitamin D-fortified yogurt on serum lipids (TG and HDL-C) compared with plain yogurt. ${ }^{25}$ Similarly, the effect of vitamin D on LDL-C and TC/HDL-C was evident and significant in combined vitamin $\mathrm{D}$ and calcium administration in Tabesh et al's trial. ${ }^{26}$

In Ramiro-Lozano and Calvo-Romero's study, ${ }^{35}$ vitamin D was given to 41 diabetic patients with vitamin $\mathrm{D}$ deficiency and insufficiency. All patients achieved a serum level of $25(\mathrm{OH}) \mathrm{D}>20 \mathrm{ng} / \mathrm{mL}$ and 25 patients $(89.3 \%)>30 \mathrm{ng} / \mathrm{mL}$. There was a significant reduction in TC, a trend but not a statistically significant reduction in LDL-C $(P=0.05)$ and non-HDL-C $(P=0.09)$. No change in HDL-C was obtained. Similar to our study, there was no significant change in serum calcium level.

Some authors suggest that possible extraskeletal effects of vitamin D require concentrations $>28-32 \mathrm{ng} / \mathrm{mL} .{ }^{36}$ 
Studies conducted by Breslavsky et al and Patel et al indicate that vitamin D treatment improving but not optimizing its level does not improve glycemia, insulin sensitivity, or lipid profile. ${ }^{37,38}$ Other studies about vitamin D replacement in diabetic patients in the absence of deficiency showed no significant effect on serum lipids. ${ }^{23}$

Reduction of serum creatinine in our study indicates a positive effect of vitamin D on kidney function in diabetic patients even with normal baseline. In support of our study, de Boer et al found a strong association between low serum vitamin D and the risk of loss of kidney function, as calculated by estimated glomerular filtration rate (eGFR) in people with normal kidney. Over 4 years of follow-up, each $10 \mathrm{ng} / \mathrm{mL}$ lower 25(OH)D was associated with a $25 \%$ greater risk of rapid eGFR loss. When compared with $25(\mathrm{OH}) \mathrm{D}$ concentration of $\geq 30 \mathrm{ng} / \mathrm{mL}, 25(\mathrm{OH}) \mathrm{D}$ concentration $<15 \mathrm{ng} / \mathrm{mL}$ was associated with a $68 \%$ greater adjusted risk of rapid eGFR loss. The association of lower $25(\mathrm{OH}) \mathrm{D}$ concentration with eGFR loss was strongest among participants with diabetes. ${ }^{39}$

Renin-angiotensin-aldosterone system (RAAS) activation and hyperfiltration characterize diabetic kidney disease. ${ }^{40}$ In animal models, vitamin D suppresses the RAAS, especially in hyper-reninemic states such as treatment with angiotensin receptor blockers, which are in common use in diabetic patients. ${ }^{41}$ Clinical trials also indicate that paricalcitol lowers albuminuria in diabetic kidney disease. ${ }^{42}$

\section{Conclusion}

Our study is the second of its type in the Saudi population with results indicating that vitamin $\mathrm{D}$ replenishment can improve glucose homeostasis and cholesterol profile in diabetic patients. It also indicates the high prevalence of this vitamin deficiency in both men and women. Through this study and other studies in this region, we can conclude the importance of vitamin D screening and replacement in this population. Because our study is small and did not include a placebo group, we recommend controlled trials with eminent number of patients of this ethnicity to reach a solid conclusion. Our results also suggest that future vitamin $\mathrm{D}$ intervention studies might target persons with diabetes for reasons beyond the effects on blood sugar and lipids such as the effects on kidney function in the absence of apparent kidney disease.

\section{Disclosure}

The authors report no conflicts of interest in this work.

\section{References}

1. Zittermann A. Vitamin D and disease prevention with special reference to cardiovascular disease. Prog Biophys Mol Biol. 2006;92(1):39-48.
2. Alvarez JA, Ashraf A. Role of vitamin D in insulin secretion and insulin sensitivity for glucose homeostasis. Int J Endocrinol. 2010; 2010:351385.

3. Foss YJ. Vitamin D deficiency is the cause of common obesity. Med Hypotheses. 2009;72(3):314-321.

4. Palomer X, González-Clemente JM, Blanco-Vaca F, Mauricio D. Role of vitamin D in the pathogenesis of type 2 diabetes mellitus. Diabetes Obes Metab. 2008;10(3):185-197.

5. Maestro B, Molero S, Bajo S, Dávila N, Calle C. Transcriptional activation of the human insulin receptor gene by 1,25-dihydroxyvitamin D. Cell Biochem Funct. 2002;20(3):227-232.

6. Zittermann A, Schleithoff SS, Koerfer R. Putting cardiovascular disease and vitamin D insufficiency into perspective. Br J Nutr. 2005; 94(4):483-492.

7. Saedisomeolia A, Taheri E, Djalali M, Moghadam AM, Qorbani M. Association between serum level of vitamin $\mathrm{D}$ and lipid profiles in type 2 diabetic patients in Iran. J Diabetes Metab Disord. 2014;13(1):7.

8. Jorde R, Grimnes G. Vitamin D and metabolic health with special reference to the effect of vitamin D on serum lipids. Prog Lipid Res. 2011; 50(4):303-312.

9. Wang H, Xia N, Yang Y, Peng D. Influence of vitamin D supplementation on plasma lipid profiles: a meta-analysis of randomized controlled trials. Lipids Health Dis. 2012;11:42.

10. Vogeser M. Apostolos Kyriat Soulis: candidate reference. Methodology for the quantification of circulating 25-hydroxy vitamin D3 by liquid chromatography-tandem mass spectrometry. Clin Chem. 2004; 50:1415-1517.

11. Fossati P, Prencip L. Serum triglycerides determined colorimetrically with an enzyme that produces hydrogen peroxidase. Clin Chem. 1982; 28(10):2077-2080.

12. Hino K, Nakamura M, et al. A new method for the homogenous assay of serum HDL-cholesterol. Clin Chem. 1996;42:5299.

13. Warnick GR, Knopp RH, Fitzpatrick V, Branson L. Estimating lowdensity lipoprotein cholesterol by the Friedewald equation is adequate for classifying patients on the basis of nationally recommended cutpoints. Clin Chem. 1990;36(1):15-19.

14. Trinder P. Determination of glucose in blood using glucosenoxidase with an alternative oxygen acceptor. Ann Clin Biochemis. 1969;6:24-27.

15. Genc S, Omer B, GurdolF, Aycan-Ustyol E, Ince N, BalF. Evaluation of turbidimetricinhibition immunoassay(TINIA) and HPLCmethods forglycated haemoglobin determination. J Clin Lab Anal. 2012;26(6):481-485.

16. Dawson-Hughes B, Heaney RP, Holick MF, Lips P, Meunier PJ, Vieth R. Estimates of optimal vitamin D status. Osteoporos Int. 2005;16(7): 713-716.

17. Muniyappa R, Lee S, Chen H, Quon MJ. Current approaches for assessing insulin sensitivity and resistance in vivo: advantages, limitations, and appropriate usage. Am J Physiol Endocrinol Metab. 2008; 294(1):15-26.

18. Al-Daghri NM, Alkharfy KM, Al-Othman A, et al. Vitamin D supplementation as an adjuvant therapy for patients with T2DM: an 18-month prospective interventional study. Cardiovasc Diabetol. 2012;11:85.

19. Sabherwal S, Bravis V, Devendra D. Effect of oral vitamin D and calcium replacement on glycaemic control in South Asian patients with type 2 diabetes. Int J Clin Pract. 2010;64(8):1084-1089.

20. Tishkoff DX, Nibbelink KA, Holmberg KH, Dandu L, Simpson RU. Functional vitamin D receptor (VDR) in the t-tubules of cardiac myocytes: VDR knockout cardiomyocyte contractility. Endocrinology. 2008;149(2):558-564.

21. Soric MM, Renner ET, Smith SR. Effect of daily vitamin D supplementation on $\mathrm{HbA} 1 \mathrm{c}$ in patients with uncontrolled type 2 diabetes mellitus: a pilot study. J Diabetes. 2012;4(1):104-105.

22. von Hurst PR, Stonehouse W, Coad J. Vitamin D supplementation reduces insulin resistance in South Asian women living in New Zealand who are insulin resistant and vitamin D deficient - a randomised, placebo-controlled trial. Br J Nutr. 2009;103(4):549-555. 
23. Talaei A, Mohamadi M, Adgi Z. The effect of vitamin D on insulin resistance in patients with type 2 diabetes. Diabetol Metab Syndr. 2013;5(1):8.

24. Sugden JA, Davies JI, Witham MD, Morris AD, Struthers AD. Vitamin D improves endothelial function in patients with Type 2 diabetes mellitus and low vitamin D levels. Diabet Med. 2008;25(3):320-325.

25. Shab-Bidar S, Neyestani TR, Djazayery A, et al. Regular consumption of vitamin D-fortified yogurt drink (Doogh) improved endothelial biomarkers in subjects with type 2 diabetes: a randomized double-blind clinical trial. BMC Med. 2011;9:125.

26. Tabesh M, Azadbakht L, Faghihimani E, Tabesh M, Esmaillzadeh A. Effects of calcium-vitamin D co-supplementation on metabolic profiles in vitamin $\mathrm{D}$ insufficient people with type 2 diabetes: a randomised controlled clinical trial. Diabetologia. 2014;57(10): 2038-2047.

27. Chiu KC, Chu A, Go VL, Saad MF. Hypovitaminosis D is associated with insulin resistance and beta cell dysfunction. Am J Clin Nutr. 2004; 79(5):820-825.

28. Simpson RU, Thomas GA, Arnold AJ. Identification of 1,25dihydroxyvitamin D3 receptors and activities in muscle. J Biol Chem. 1985;260(15):8882-8891.

29. Maestro B, Dávila N, Carranza MC, Calle C. Identification of a Vitamin D response element in the human insulin receptor gene promoter. J Steroid Biochem Mol Biol. 2003;84(2-3):223-230.

30. Cohen-Lahav M, Douvdevani A, Chaimovitz C, Shany S. The antiinflammatory activity of 1,25-dihydroxyvitamin D-3 in macrophages. J Steroid Biochem Mol Biol. 2007;103:558-562.

31. Ojuka EO. Role of calcium and AMP kinase in the regulation of mitochondrial biogenesis and GLUT4 levels in muscle. Proc Nutr Soc. 2004;63(2):275-278.

32. Dunlop TW, Väisänen S, Frank C, Molnár F, Sinkkonen L, Carlberg C. The human peroxisome proliferator-activated receptor $\delta$ gene is a pri-

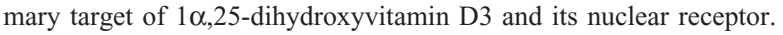
J Mole Biol. 2005;349(2):248-260.
33. Kumar S, Davies M, Zakaria Y, et al. Improvement in glucose tolerance and beta-cell function in a patient with vitamin $\mathrm{D}$ deficiency during treatment with vitamin D. Postgrad Med J. 1994;70(824):440-443.

34. Jafari T, Fallah AA, Barani A. Effects of vitamin D on serum lipid profile in patients with type 2 diabetes: a meta-analysis of randomized controlled trials. Clin Nutr. 2016;35(6):1259-1268.

35. Ramiro-Lozano JM, Calvo-Romero JM. Effects on lipid profile of supplementation with vitamin D in type 2 diabetic patients with vitamin D deficiency. Ther Adv Endocrinol Metab. 2015;6(6):245-248.

36. Hossein-nezhad A, Spira A, Holick MF. Influence of vitamin D status and vitamin D3 supplementation on genome wide expression of white blood cells: a randomized double-blind clinical trial. PLoS One. 2013;8(3):e58725.

37. Breslavsky A, Frand J, Matas Z, Boaz M, Barnea Z, Shargorodsky M. Effect of high doses of vitamin D on arterial properties, adiponectin, leptin and glucose homeostasis in type 2 diabetic patients. Clin Nutr. 2013;32(6): 970-975.

38. Patel P, Poretsky L, Liao E. Lack of effect of subtherapeutic vitamin D treatment on glycemic and lipid parameters in Type 2 diabetes: a pilot prospective randomized trial. J Diab. 2010;2(1):36-40.

39. de Boer IH, Katz R, Chonchol M, et al. Serum 25-hydroxyvitamin D and change in estimated glomerular filtration rate. Clin J Am Soc Nephrol. 2011;6(9):2141-2149.

40. Li YC, Kong J, Wei M, Chen ZF, Liu SQ, Cao LP. 1,25-Dihydroxyvitamin $\mathrm{D}(3)$ is a negative endocrine regulator of the renin-angiotensin system. J Clin Invest. 2002;110(2):229-238.

41. Zhang Z, Zhang Y, Ning G, Deb DK, Kong J, Li YC. Combination therapy with AT1 blocker and vitamin D analog markedly ameliorates diabetic nephropathy: blockade of compensatory renin increase. Proc Natl Acad Sci U S A. 2008;105(41):15896-15901.

42. de Zeeuw D, Agarwal R, Amdahl M, et al. Selective vitamin D receptor activation with paricalcitol for reduction of albuminuria in patients with type 2 diabetes (VITAL study): a randomised controlled trial. Lancet. 2010;376(9752):1543-1551.
Therapeutics and Clinical Risk Management

\section{Publish your work in this journal}

Therapeutics and Clinical Risk Management is an international, peerreviewed journal of clinical therapeutics and risk management, focusing on concise rapid reporting of clinical studies in all therapeutic areas, outcomes, safety, and programs for the effective, safe, and sustained use of medicines. This journal is indexed on PubMed Central, CAS,

\section{Dovepress}

EMBase, Scopus and the Elsevier Bibliographic databases. The manuscript management system is completely online and includes a very quick and fair peer-review system, which is all easy to use. Visit $\mathrm{http}: / /$ www.dovepress.com/testimonials.php to read real quotes from published authors. 